\title{
Pulmonary tuberculosis mimicking numerous lung metastases - a case study
}

\author{
Corresponding author: \\ Agnieszka Nowakowska-Arendt, $\mathrm{PhD}$ \\ Department of Palliative Care, \\ Nicolaus Copernicus University, Torun \\ Collegium Medicum in Bydgoszcz \\ Jagiellońska 13-15 St. \\ 85-067 Bydgoszcz, Poland \\ Tel. +48525853306 \\ E-mail: agnieszka.arendt@cm.umk.p
}

Medical Research Journal 2018; Volume 3, Number 1, 43-46 10.5603/MRJ.2018.0008

Copyright (C) 2018 Via Medica ISSN 2451-2591

\begin{abstract}
SUMMARY
Introduction. Tuberculosis is an infectious disease caused by tuberculosis bacilli. Presently, the incidence of tuberculosis is deemed low in Poland, i.e. less than 20 cases per 100,000 residents, even though this value still exceeds the EU average. Most often it affects the lungs, although it may also develop in other organs. The diagnosis is based on the positive test result of tuberculosis bacilli breeding on the Löwenstein-Jensen's egg medium. Under no circumstances may an X-ray image of the chest be construed as the ultimate diagnostic confirmation of tuberculosis, though.

Case description. The case of a 68-year old woman, put under diagnostic procedure due to an abnormal $X$-ray image of the lungs, thereby giving rise to the suspicion of numerous metastases of unknown origin, was addressed. The initially suspected neoplastic disease with lung metastases, following comprehensive pulmonary diagnostics, ultimately turned out to be pulmonary tuberculosis, despite presenting a non-characteristic, radiological image.

Conclusions. Completion of a comprehensive, differential diagnostics is always a prudent approach to be adopted, ultimately with a view to establishing the patient's status beyond a reasonable doubt. Key words: lung metastases, tuberculosis, thyroid cancer
\end{abstract}

Med Res J 2018; 3 (1): 43-46

\section{Introduction}

Tuberculosis is an infectious disease caused by tuberculosis bacilli. The diagnosis is based on the positive result of tuberculosis bacilli breeding on the Löwenstein-Jensen's egg medium. Even though the incidence of tuberculosis and attendant mortality have gradually decreased across Europe, it is still deemed a potentially lethal disease. Presently, the World Health Organization regards Poland as a country with a low incidence of tuberculosis, i.e. less than 20 cases per 100,000 residents. Not only is the low incidence of tuberculosis attributable to a consistent policy of protective vaccinations, but also to much improved living conditions, and overall quality of life, both in Poland and Europe.

Pulmonary tuberculosis makes up a vast majority of all cases diagnosed in 2016, i.e. $94.9 \%$. Its most common extra-pulmonary form is tuberculous pleurisy. Thyroid TB is deemed a very rare clinical phenomenon, even in the countries where the incidence of tuberculosis is higher than the European average. There are several dozen descriptions of thyroid TB manifestations published to date.

\section{Case report}

A 68-year old woman was admitted to the Dept. of Pulmonary Diseases in July 2014, due to an abnormal $X$-ray image of the chest, uncannily evocative of lung metastases. Four days earlier, the patient had been admitted to a consultation in the Accident and Emergency Dept., complaining of a chest pain, and a "feeling of being clogged up". The pain complaint tended to intensify upon every deep intake of breath, and had already been bothering the patient for several days. This was accompanied by exertion dyspnoea and coughing, which brought some relief, though.

Following a comprehensive diagnostic procedure, any cardiologic basis of the reported pulmonary complaints was ruled out. The patient admitted to thyroid nodular goiter in the interview, in euthyreosis at the time. She remained under periodic monitoring at the 
Endocrine Outpatients Clinic. Despite the earlier, normal laboratory results of thyroid function, the patient reported occasional "heart palpitations" and anxiety, although she did not seek any consultations with her doctor. Nor did she remain on permanent pharmacotherapy. She worked as a hairdresser; a non-smoker. During her hospital consultation, several exams were completed, inter alia, an X-ray examination of the chest which revealed numerous round shadows (more numerous on the right-hand side), sized up to $20 \mathrm{~mm}$, subsequently referred to as the suspected metastases in both lungs. Furthermore, a low density bone structure in the distal part of rib I on the right-hand side was also detected. Due to the suspicion of a disseminated neoplastic disease, the patient was issued with a referral for further pulmonary diagnostics.

The subjective examination revealed exertion dyspnoea, properly warmed-up skin covered with perspiration; in the left supraclavicular pit, a lymph node easily discernible under the touch, approx. $1.5 \mathrm{~cm}$ in diameter. In laboratory tests - elevated inflammatory markers (OB $70 \mathrm{~mm}$, CRP $24.44 \mathrm{mg} / \mathrm{l}$, without leukocytosis, with an increased percentage of granulocytes $83.7 \%$, and a reduced percentage of lymphocytes 8.5\%). The CEA and Ca125 cancer markers remained within their normal range. Indeterminable values for TSH were reported. In the thyroid and parathyroid ultrasound examination the right thyroid gland was visualized $-16 \times 18 \times 40 \mathrm{~mm}$, the left one $-24 \times 21 \times 46 \mathrm{~mm}$. In both glands, numerous, hypoechogenic, confluent, partially encapsulated nodular lesions with calcifications in the middle of up to $13 \mathrm{~mm}$, and the single anechoic ones of up to $10 \mathrm{~mm}$, were encountered. No enlarged lymph nodes were encountered on the neck, though. In both supraclavicular pits, the lymph nodes were visible, $2.6 \mathrm{~mm}$ long along the short axis. Following a consultation with the endocrinologist, treatment with a full-dose antithyroid drug was commenced.

Numerous meta foci in both lungs, up to approx. $22 \mathrm{~mm}$ in segment 1 of the right lung were revealed in the chest CT. No enlarged mediastinal nodes and cavities were detected. Adrenal glands were found not enlarged, no changes within the bone structure were observed, either (Figure 1).

In view of a suspected neoplastic disease of an unknown origin, the aspiration biopsy of one of the lesions located in the left lung was carried out twice. An enlarged supraclavicular node was also taken for histopathological examination (under local anesthesia). Cytological examination of the material acquired through the biopsy of the lesion in the left lung, revealed necrotic masses, numerous macrophages with epithelial-cellular appearance, rather numerous lymphocytes and neutrophils. Histopathologic examination did not reveal any neoplastic changes. Non-specific reactive

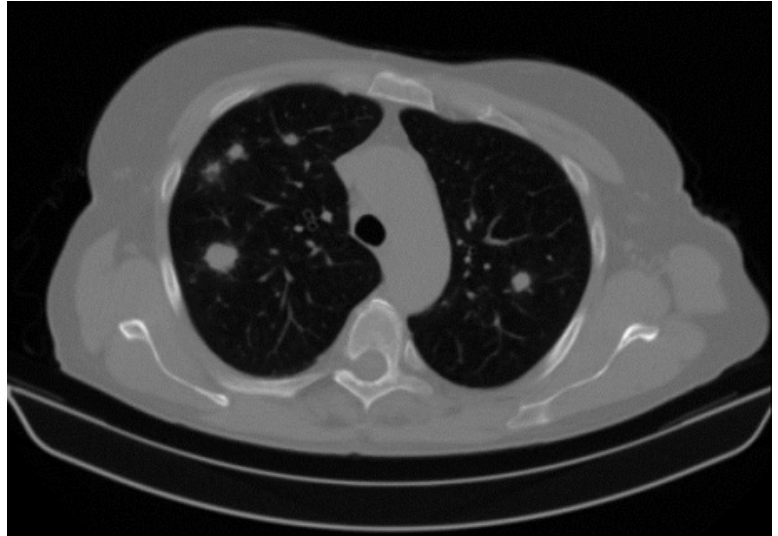

Figure 1. Numerous rounded changes in both pulmonary fields (Chest CT scan of July 2014).

lymphadenitis (lymphadenitis non-specific reactiva) was detected, though. In view of numerous hypoechogenic changes in the thyroid gland, as revealed through an ultrasound exam, tumour-like in appearance in both thyroid lobes, non-assayable TSH value, and numerous rounded lesions in the lungs, a suspicion of thyroid cancer with pulmonary metastases was then hypothesized.

Published reports on the pathologies in the thyroid gland were comparatively assessed, and the histopathological image of the lymph node tissue, as collected through biopsy, was found to correspond to the thyroid biopsy results described by A. Ortiz-Flores et al (1), who discussed the case of thyroid TB. Respiratory diagnostics were then pursued further. The differential diagnosis prompted the suspicion of an atypical manifestation of tuberculosis. The bronchofiberoscopic examination revealed a slightly reddened mucous membrane within the entire bronchial tree, which may have accounted for an inflammation. The bacteriological examination of bronchial lavage neither revealed any tuberculosis bacilli, nor their DNA, while the culture breeding result also proved negative. Cytological examination revealed a mucous content with numerous neutrophils and macrophages. During further diagnostics, the following results were obtained: QuantiFERON TB, positive, $8.1 \mathrm{lU} / \mathrm{ml}$ ( $>=0.5 \mathrm{IU} / \mathrm{ml}$ positive result). Following endoscopic examination of the respiratory tracts, imaging exams, histopathological assessment, and the QuantiFeron result, pulmonary tuberculosis was ultimately diagnosed.

Despite initial suspicion of a neoplastic disease with lung metastases, following comprehensive pulmonary diagnostics, pulmonary tuberculosis was ultimately established as the actual cause, despite its non-characteristic radiological image. The present clinical case only goes to show that a comprehensive diagnosis is always a prudent option, as it may well prove instrumental in contributing to a totally unexpected, definitive diagnosis. 


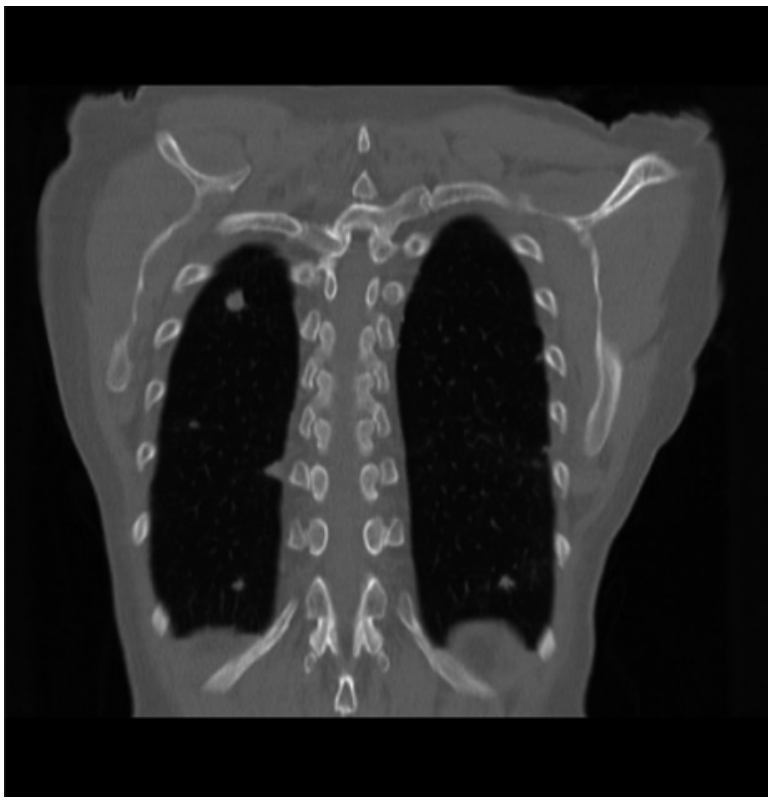

Figure 2. Numerous rounded changes in both pulmonary fields (Chest CT scan of July 2014).

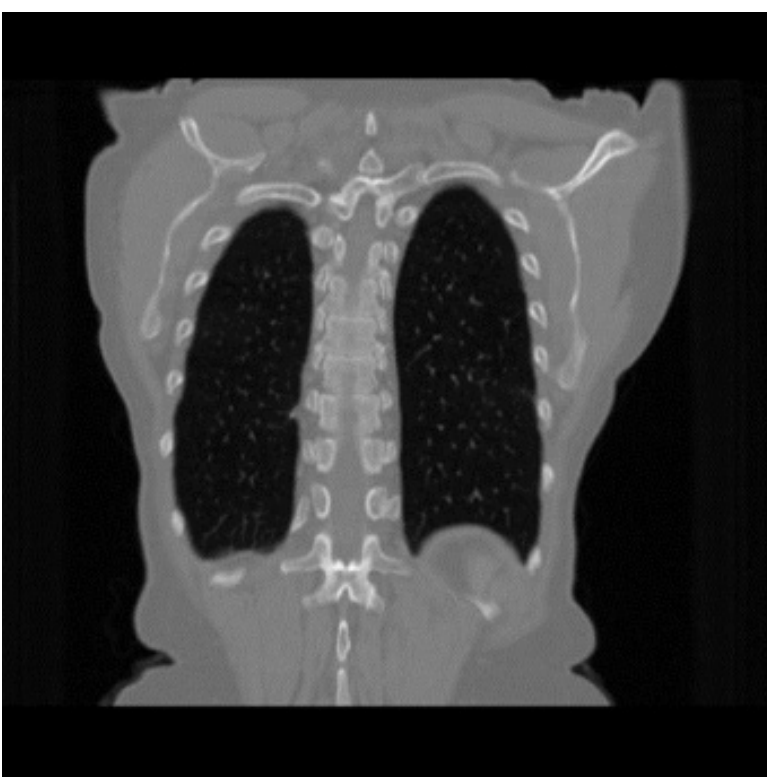

Figure 3. Chest CT scan of Dec., 2014

Anti-tuberculosis treatment was promptly commenced, in line with all applicable standards. The patient initially received a combination treatment, i.e. rifampicin $600 \mathrm{mg} / \mathrm{d}$ + isoniazid $300 \mathrm{mg} / \mathrm{d}$ + pyrazynamid $1500 \mathrm{mg} / \mathrm{d}$. Introduction of ethambutol was delayed in view of the need for an ophthalmological consultation, hence it was incorporated into the treatment management upon the 21st day, at the dose of $1250 \mathrm{mg} / \mathrm{d}$.
The patient remained on the full treatment for two months, followed by a combined rifampicin and isoniazid therapy (at $600 \mathrm{mg} / \mathrm{day}$ and $300 \mathrm{mg} / \mathrm{d}$ dose, respectively) for the following four months. The patient attended regular check-ups in the in-house out-patient clinic; the last one completed in June 2017. The treatment was tolerated very well, with no adverse reactions whatsoever. After 6 months, the clinical symptoms, e.g. shortness of breath, and coughing, were successfully resolved. An X-ray image of the lungs revealed gradual resorption of the nodular changes in the pulmonary parenchyma (Figure 3). Endocrine treatment has been continuing under the supervision of the in-house endocrine outpatient clinic.

Within a year of the commencement of anti-tuberculosis treatment, a slight reduction in the number of leukocytes was observed (WBC 3.76 * $10 \wedge 3$ / ul - WBC: $3.45 * 10 \wedge 3$ [mu] I [4.0 - 10.0]) and neutrophil granulocytes (neutra: $2.1110 \wedge 3$ / Ul neut: $1.9810 \wedge 3 /$ uL [2.50 - 5.0]). The patient was advised to attend a haematological consultation, which she failed to complete. The last check-up of morphotic parameters, carried out on Dec. 12, 2016, yielded no deviations from the reference values.

\section{Discussion}

Over the recent years, both the incidence of tuberculosis and the attendant mortality rate has gradually been decreasing in Poland, even though still remaining higher than the European average. Presently, Poland is officially rated among the countries with a low incidence of tuberculosis. By far the most common form of tuberculosis is its pulmonary manifestation. Patients suffering exclusively from the extra-pulmonary tuberculosis accounted merely for $5.1 \%$ (i.e. 328 cases) of all cases registered throughout the country in 2016 . Tuberculous pleurisy proved its most frequent manifestation. Not a single case of thyroid TB was registered in 2016 in Poland (2).

Over the last fifteen years, several dozen cases of thyroid TB have been published. In some cases, the diagnosis was made only against the post mortem exam (3). It is still pending credible explanation, though, why thyroid TB tends to occur sporadically. This might likely be down to a diversity of attendant pathomechanisms, e.g. bactericidal properties of the colloid, increased vascular supply and oxygenation of the thyroid gland, as well as high iodine concentration (4-5). A typical manifestation of tuberculosis in the lungs is not hard to be detected, even though occasionally it may pose a bona fide diagnostic challenge. The most common cause of the rounded shadows encountered on the $\mathrm{X}$-ray image of the lungs is a cancerous disease. Such 
a manifestation of pulmonary tuberculosis is very rare, indeed (6-9). It should nevertheless be always borne in mind that it is a potentially promising diagnostic avenue to be pursued, if only to save the patient going through a number of unnecessary, often invasive diagnostic procedures. In the Authors' opinion, the present clinical case seems to demonstrate persuasively enough that completion of a comprehensive, differential diagnostics is always a prudent approach to be adopted, ultimately with a view to establishing the patient's status beyond a reasonable doubt.

\section{References}

1. Ortiz-Flores A, Gioia F, Montánez-Fernández L, et al. Incidental finding of a primary thyroid tuberculosis. Oxf Med Case Reports. 2017; 2017(6): omx022, doi: 10.1093/omcr/omx022, indexed in Pubmed 28580157
2. Korzeniewska-Koseła M. Tuberculosis in Poland 2016* Gruźlica w Polsce w 2016 roku* — Instytut Gruźlicy i Chorób Płuc: Warszawa.

3. Bulbuloglu E, Ciralik H, Okur E, et al. Tuberculosis of the thyroid gland: review of the literature. World J Surg. 2006; 30(2): 149-155, doi: 10.1007/s00268-005-0139-1, indexed in Pubmed: 16425087.

4. Lourtet-Hascoet J, Le Grusse J, Fontaine S, et al. Thyroid tuberculosis: a new case and review of the literature. Ann Endocrinol (Paris). 2015; 76(5): 635-637, doi: 10.1016/j.ando.2015.07.934, indexed in Pubmed: 26602881

5. Hernández MC, Hoyos EG, Rojas EA, et al. Tuberculosis tiroidea: causa excepcional de bocio compresivo. Endocrinología y Nutrición. 2013; 60(8): e11-e13, doi: 10.1016/j.endonu.2012.10.005.

6. Pilaniya V, Gera K, Kunal S, et al. Pulmonary tuberculosis masquerading as metastatic lung disease. Eur Respir Rev. 2016; 25(139): 97-98, doi: 10.1183/16000617.00002315, indexed in Pubmed: 26929427.

7. Ariyürek $\mathrm{MO}$, Karçaaltincaba M, Demirkazik FB, et al. Bilateral multiple pulmonary tuberculous nodules mimicking metastatic disease. Eur $\mathrm{J}$ Radiol. 2002; 44(1): 33-36, indexed in Pubmed: 12350408.

8. Paci M, Cavazza A, Ferrari G, et al. Image of the month. Pulmonary tuberculosis mimicking lung metastasis. J Thorac Oncol. 2008; 3(6): 660-661, doi: 10.1097/JTO.0b013e3181757ad4, indexed in Pubmed: 18520809.

9. Kant S, Saheer S, Prakash V, et al. Bilateral nodular pulmonary tuberculomas simulating metastatic disease. BMJ Case Rep. 2011; 2011, doi: 10.1136/bcr.11.2010.3539, indexed in Pubmed: 22701010 